\title{
Association between CD127 Gene Polymorphism and Susceptibility to Multiple Sclerosis
}

\author{
Fereshteh Alsahebfosoul ${ }^{1,6 *}$, Rasoul Salehi' ${ }^{2}$, Masoud Etemadifar $^{3,5}$, Ahmad Zavaran Hosseini $^{4}$ \\ Mohammad Kazemi², Mehrdad Farokhi ${ }^{5,6}$, and Mohadaseh Behjati ${ }^{5}$ \\ ${ }^{1}$ Immunology Department, Faculty of medicine, Isfahan University of medical sciences, Isfahan, Iran \\ ${ }^{2}$ Molecular biology and genetics Department, Faculty of medicine, Isfahan University of medical sciences, Isfahan, Iran \\ ${ }^{3}$ Neurology Department, Faculty of medicine, Isfahan University of medical sciences, Isfahan, Iran \\ ${ }^{4}$ Immunology Department, Faculty of medicine, Tarbiat Modares University, Tehran, Iran \\ ${ }^{5}$ Faculty of medicine, Isfahan University of medical sciences, Isfahan, Iran \\ ${ }^{6}$ Isfahan multiple sclerosis Research Center, Isfahan, Iran
}

Received: July 27, 2016; Accepted: September 26, 2016; Published: September 30, 2016

*Corresponding author: Fereshteh Alsahebfosoul, Immunology Department, Faculty of medicine, Isfahan University of medical sciences, Isfahan, Iran, E-mail: alsahebfosoul@med.mui.ac.ir

\begin{abstract}
Background: Multiple Sclerosis (MS) is an autoimmune disease of Central Nervous System (CNS) which is supposed the interaction of genetic and environmental factors have a role in its pathogenesis. Previous studies have described association between some single nucleotide polymorphisms of IL7Ra gene and risk of MS. Rs6897932 SNP is located in coding region of this gene is associated with increased level of soluble form of IL7Ra and so may be correlated with development of MS.
\end{abstract}

Methods: In this case-control study, DNA from peripheral blood of 200 Relapsing Remitting Multiple Sclerosis (RRMS) patient of Isfahan Multiple Sclerosis Society (IMSS) is extracted and then allele frequencies and genotyping were performed using HRM-PCR method.

Results: Our results showed that there is no significant difference between RRMS patients and controls with respect to the distribution of IL7Ra gene polymorphism $(P=0.57, \mathrm{OR}=1.16)$. Further analysis after adjusting for age and sex has displayed similar results.

Conclusion: We could not find association between CD127 gene polymorphism and risk of MS; however, more studies with larger sample sizes in different ethnical populations are needed to demonstrate role of this SNP in pathogenesis of MS.

Keywords: Multiple sclerosis; CD127; rs6897932; IL7Ra

\section{Introduction}

Multiple Sclerosis (MS) is a chronic inflammatory disease of Central Nervous System (CNS) that affects about 2.5 million individuals worldwide and has been known as the most common debilitating neurological disease in young adults.MS is an autoimmune disease characterized with myelin loss and axonal damages which lead to neurological symptoms including: impairment of vision, movement, and balance that vary widely in different patients. It is supposed that MS is caused by the interaction of genetic and environmental factors, for example, infection agents [1]. Although many investigations have been carried out to indicate roles of various factors in pathogenesis of MS, exact etiology of MS is not fully known. Investigations of genetic risk factors have been provided evidences for association between susceptibility to genetically autoimmune diseases such as MS and polymorphisms in the Human Leukocyte Antigen (HLA) complex especially, DRB1*15:01 and DQB1*06:02 on chromosome 6p21 [2]. Recently other candidate gene approaches or Genome wide association studies suggested new loci outside HLA region such as cytokines and their receptors genes as suspected genes for further investigations, although these associations could not be demonstrated by other studies which their result varied widely in different populations. It has been shown that IL-7 is an essential cytokine for development and maturation of T-cells and also has a role in proliferation and function of mature T-cells but previous studies could not find exact mechanism of IL-7 production completely [3]. IL-7R is a heterodimer molecule which is consisted of $\alpha$-chain or IL-7R $\alpha$ and $\Upsilon$-chain or IL-7RY. Furthermore, IL-7R $\alpha$ is a component of the receptor for Thymic Stromal Lymphopoietin (TSLP) which is produced by Hassall's corpuscles and is implicated in induction of thymocytes differentiation into Treg [4]. Rs 6897932 is a single nucleotide polymorphism in IL7Ra gene with a vital role in Multiple Sclerosis, and the major allele $\mathrm{C}$ enhancement mutation of exon 6 and cause to increase production the soluble of IL7Ra. IL7Ra is involved in the improvement and homeostasis of B and T-lymphocytes [5].

The (C) allele of rs6897932 synthesis the amino acid threonine greater than isoleucine at position 244that associated with an increased risk of multiple sclerosis. The $\mathrm{C}$ allele is the most common in all recognized population and influences the ratios membrane and soluble isoforms of the gene and $\mathrm{T}$ allele is protective. Under the influence reaction Interleukin-7 receptor alpha with IL-7 a, formed signaling complex, that is important to maintain memory $\mathrm{T}$ cells, development, proliferation and survival of $\mathrm{B}$ and $\mathrm{T}$ lymphocytes, especially $\mathrm{CD} 4+$ positive $\mathrm{T}$ that seen in lesions of MS patients. 
AlthoughIL-7R $\alpha$ or CD-127 SNP has been investigated in previous studies, their result were controversial [6,7] and it has also been shown that frequencies of SNP polymorphisms in genetically autoimmune diseases such as MS vary in different ethnic populations in some studies [8]. So in this study we aimed to investigate association between different genotypes of rs6897932 SNP with risk of MS in Iranian population residing in the province of Isfahan, Iran.

\section{Materials and Methods}

This case-control study was conducted on two hundred MS patients of Isfahan Multiple Sclerosis Society (IMSS) who were all Iranian and residing in Isfahan, central province of Iran and two hundred healthy subjects of Isfahan Transfusion Organization (control group) who were matched for age and sex. All of MS patients were Relapsing Remitting (RR) type and had diagnosed with clinically definite MS according to McDonald's criteria [9] and also their Expanded Disability Status Scale (EDSS) were used to determine degree of disability in RRMS patients [10]. The patients were at remission phase of disease. Protocol of this study was approved by ethical committee of Isfahan University of medical sciences and also their informed consensuses were obtained from all MS patients and healthy control group.

Peripheral blood samples were collected from RRMS patients and healthy controls in tubes containing EDTA (Ethylene diamine tetra acetic acid ) as anticoagulant and then DNA was extracted by QIAamp DNA Blood Midi Kit' (Quiagen, Hilden, Germany) according to themanufacturer'sinstructions. To determine quality of extracted DNA, agarose gel electrophoresis was used by UV absorption at 260 and $280 \mathrm{~nm}$. The CD127 SNP results in the replacement of C to $\mathrm{T}$ in the coding region of exon 6. Real-time PCR was performed using forward primer (5'-CTTCAAGTGGCAGATGCTCTG) and reverse primer (3'-CCCACACAATCACCCTCTTTAT) to amplify the DNA region containing the CD127 SNP. Real-time-PCR was implemented under the following condition: $95^{\circ} \mathrm{C}$ for 5 minutes followed by 40 cycles of $95^{\circ} \mathrm{C}$ for 10 seconds, $55^{\circ} \mathrm{C}$ for 20 seconds, and $72^{\circ} \mathrm{C}$ for 10 seconds. High resolution melting curve (HRM) analysis was used for SNP genotyping, so we raised temperature gradually from $65^{\circ} \mathrm{C}$ to $95^{\circ} \mathrm{C}$ within 2 minutes.

Data were analyzed using the SPSS program (version 20; SPSS Inc., Chicago, USA).Chi square test was employed for HardyWeinberg equilibrium and comparison of genotype and allele frequencies. Furthermore, odds ratio and P-value were calculated using logistic regression. Results have been reported as a mean $( \pm$ SD) and number (percent). All tests were two-tailed, and a $P$-value of $<0.05$ was considered.

\section{Results}

This study population consisted of 200 RRMS patients (40 males and 160 females) and 200 healthy subjects (37 men and 163 women). Mean age of patients and controls were $32.35 \pm 7.50$, $31.22 \pm 8.49$, respectively. No statistically differences were found between patients and controls concerning age and gender $(P>$ 0.05).Clinical and paraclinical features of patients and controls who participated in this study are summarized in Table 1.
Frequency of C/T and T/T genotypes of rs6897932 SNP were $26 \%$ and $74 \%$ in RRMS patients and $39 \%$ and $71 \%$ in controls, respectively and also it has been shown that frequency of alleles in patients and controls were close to each other. In addition, statistical analysis using Fisher test could not find significant association between patients and controls with respect to the distribution of genotypes (0.576) and alleles (0.772), suggesting no association between CD127 gene polymorphism and susceptibility to MS. Allele and genotype distribution of rs6897932 SNP in cases and controls are shown in Table 2.

Further analysis revealed that EDSS $(P=0.476)$ has no significant association with type of genotypes in the treated patients. On the other hand, the duration of remission after treatment is no differences in genotype distribution between the two patient subgroups $(P=0.385)$ as well as it was revealed that treatment for more than one year, CT genotypesis less prevalentthan CC (Table 3).

\section{Discussion}

In this study, we considered distribution of different genotypes of CD127 gene among RRMS patients in comparison with healthy subjects and association of rs6897932 SNP with risk of MS disease. Our results showed that there is no significant differences between cases and controls with respect to the

Table 1: Demographic and clinical features of cases and controls

\begin{tabular}{|c|c|c|c|c|}
\hline \multicolumn{2}{|l|}{ Variables } & $\operatorname{Case}(n=200)$ & $\begin{array}{l}\text { Control }(n= \\
200)\end{array}$ & P-Value \\
\hline \multicolumn{2}{|l|}{ Age; year } & $31.22 \pm 8.49$ & $32.35 \pm 7.50$ & 0.159 \\
\hline \multirow{2}{*}{ Gender } & Male & $40(20 \%)$ & $37(18.55)$ & \multirow{2}{*}{0.800} \\
\hline & Female & $160(80 \%)$ & $163(81.5 \%)$ & \\
\hline \multirow{3}{*}{ Education } & Preliminary & $50(26.6 \%)$ & $51(25.8 \%)$ & \multirow{3}{*}{0.363} \\
\hline & Secondary & $75(39.9 \%)$ & $92(46.5 \%)$ & \\
\hline & University & $63(33.5 \%)$ & $55(27.8 \%)$ & \\
\hline \multirow{2}{*}{ Family status } & Single & $12(30.8 \%)$ & $49(24.6 \%)$ & \multirow{2}{*}{0.427} \\
\hline & Married & $27(69.2 \%)$ & $150(75.4 \%)$ & \\
\hline \multirow{3}{*}{ EDSS } & $\leq 1$ & $95(87.2 \%)$ & - & \multirow{3}{*}{-} \\
\hline & $(1-2)$ & $9(8.3 \%)$ & - & \\
\hline & $>2$ & $5(4.6 \%)$ & - & \\
\hline \multirow{2}{*}{ Treatment } & Treated & $115(57.5 \%)$ & - & \\
\hline & Untreated & $85(42.5 \%)$ & - & \\
\hline \multirow{2}{*}{$\begin{array}{l}\text { Duration of } \\
\text { remission after } \\
\text { treatment }\end{array}$} & $\leq 1$ & $51(44.3 \%)$ & - & \multirow[b]{2}{*}{-} \\
\hline & $>1$ & $64(55.7 \%)$ & - & \\
\hline
\end{tabular}

Table2: Allele and genotype distribution of rs6897932 SNP in cases and controls

\begin{tabular}{|l|l|l|l|l|l|}
\hline $\begin{array}{l}\text { Allele and } \\
\text { genotype }\end{array}$ & \multicolumn{2}{l|}{ Case(n=200) } & $\begin{array}{l}\text { Control(n = } \\
\text { 200) }\end{array}$ & $\begin{array}{l}\text { OR(95\% } \\
\text { CI) }\end{array}$ & P-Value \\
\hline $\begin{array}{l}\text { Polymorphism } \\
\text { frequency }\end{array}$ & CC & 148(74\%) & 142(71\%) & $1.16(0.74-$ & 0.576 \\
\hline CT & $52(26 \%)$ & $58(29 \%)$ & $1.80)$ & \\
\hline $\begin{array}{l}\text { Allele } \\
\text { frequency }\end{array}$ & C & $174(87 \%)$ & $171(85.5 \%)$ & $1.13(0.64-$ & 0.772 \\
\hline & T & $26(13 \%)$ & $29(14.5 \%)$ & $2.01)$ & \\
\hline
\end{tabular}

Data shown n (\%), OR (95\% CI) : Odd Ratio (95\% Confidence Interval) 
Table 3: Association between EDSS, duration of remission after treatment, and treatment with types of genotypes

\begin{tabular}{|c|c|c|c|c|c|}
\hline \multirow{2}{*}{\multicolumn{2}{|c|}{ Variables }} & \multicolumn{2}{|l|}{ Genotype } & \multirow{2}{*}{$\begin{array}{l}\text { OR }(95 \% \\
\text { CI) }\end{array}$} & \multirow{2}{*}{ P-Value } \\
\hline & & CC & CT & & \\
\hline \multirow{3}{*}{ EDSS } & $\leq 1$ & 73(85.9\%) & $22(91.7 \%)$ & \multirow{3}{*}{$\begin{array}{l}0.583(0.36- \\
0.94)\end{array}$} & \multirow{3}{*}{0.476} \\
\hline & $(1-2)$ & $7(8.2 \%)$ & $2(8.2 \%)$ & & \\
\hline & $>2$ & $5(5.9 \%)$ & $0(0 \%)$ & & \\
\hline \multirow{2}{*}{$\begin{array}{l}\text { Duration of } \\
\text { remission } \\
\text { after } \\
\text { Treatment }\end{array}$} & $\leq 1$ & $29(34.53 \%)$ & $14(45.17 \%)$ & \multirow[b]{2}{*}{$\begin{array}{l}0.640(0.277- \\
1.480)\end{array}$} & \multirow[b]{2}{*}{0.385} \\
\hline & $>1$ & $55(65.47 \%)$ & $17(54.83 \%)$ & & \\
\hline \multirow{2}{*}{ Patients } & Treated & $84(73 \%)$ & $31(27 \%)$ & \multirow{2}{*}{$\begin{array}{l}1.12(0.59- \\
2.13)\end{array}$} & \multirow{2}{*}{0.747} \\
\hline & Untreated & $64(75.29 \%)$ & $21(24.71 \%)$ & & \\
\hline
\end{tabular}

Data shown n (\%), OR (95\% CI) : Odd Ratio (95\% Confidence Interval)

distribution of IL7Ra, suggesting no association between increased risk of MS and CD127 gene polymorphism.While analysis of Zhang 's study on a European subgroup showed an association between IL7R with susceptibility to MS for the C-allele and the C/C genotype [11].

In a similar study, Iabayyan, et al. [7] investigated association between susceptibility to MS and two SNPs related to IL-7R $\alpha$ gene including rs6897932 and rs11567685 SNP in 200 MS patients and 200 healthy controls and they found that in Jordanian population there is no difference between controls and patients in terms of frequencies of rs6897932SNP $(P=0.583)$. On the other hand, analysis of rs11567685 which is located in the promoter region and affects gene expression showed that genetic profile was significantly different between MS patients and general population $(P=0.02)$. These data suggest that IL-7R $\alpha$ plays a biological role in MS via implicating in its expression, but not modification.

Our results are also in line with finding of Heidari, et al. [12] who earlier reported that susceptibility to MS in Iranian population is not associated with rs6897932 SNP, however, they found a significant difference between secondary progressive MS patients and controls. These failures to demonstrate association between risk of MS and CD127 gene polymorphisms might be due to low sample size of these studies, similar to our study in which we studied 200 RRMS patients. But it should be considered although low sample size can affect results of these studies, large sample sizes might be yielded similar results, as Traboulsee, et al. [13] studied IL7Ra gene polymorphism among 1,978 Canadian MS patients and they also failed to find association between this SNP and susceptibility to MS ( $p$ value $=0.07$ ), however, they found significant association between IL7R and patients who developed Progressive MS (PRMS) ( $p=0.002$; odds ratio $=0.73$ ).

Given complex etiology of MS and the role of both environmental and genetic factors and since genotype frequencies of SNP varies widely among different populations, we should consider role of different races in these controversial findings.

On the other hand, there are other studies which reported significant association between MS and rs6897932 gene polymorphism and in contrast to studies which could notbe found this association; their samples covered more diverse ethnic groups. Furthermore, investigation among them showed that except one study [14], almost all of them suggested that $C$ allele is associated with high risk of MS [7]. IL7Ra rs6897932 SNP is located in exon 6 which encodes integral membrane domain of the receptor, while rs6897932 SNP causes skipping at this site which leads to more soluble form of protein. In addition, it has been shown that being carrier of $\mathrm{C}$ allele is associated with reduced production of membrane-bound form of IL7Ra protein. This increased level of soluble form of IL7Ra causes an increase in response of $\mathrm{T}$ cells to myelin basic protein which may leads to development of MS [15].

In summary, we could not find association between CD127 gene polymorphism with risk of MS, however, more studies with larger sample sizes in different ethnical populations are needed to demonstrate role of this SNP in pathogenesis of MS.

\section{References}

1. Etemadifar M, Abtahi SH, Razmjoo H, Abtahi MA, Dehghani A, Salari M, et al. 25-hydroxyvitamin D Concentrations in Patients with Optic Neuritis as a Clinically Isolated Syndrome and Healthy Controls. Int J Prev Med. 2012;3(5):313-7.

2. Dyment DA, Herrera BM, Cader MZ, Willer CJ, Lincoln MR, Sadovnick $\mathrm{AD}$, et al. Complex interactions among MHC haplotypes in multiple sclerosis: susceptibility and resistance. Human molecular genetics. 2005;14(14):2019-26.

3. Jiang Q, Li WQ, Aiello FB, Mazzucchelli R, Asefa B, Khaled AR, et al. Cell biology of IL-7, a key lymphotrophin. Cytokine Growth Factor Rev. 2005;16(4-5):513-33.

4. Watanabe N, Wang YH, Lee HK, Ito T, Wang YH, Cao W, et al. Hassall's corpuscles instruct dendritic cells to induce CD4+CD25+ regulatory T cells in human thymus. Nature. 2005;436(7054):1181-5.

5. Zuvich RL, McCauley JL, Oksenberg JR, Sawcer SJ, De Jager PL, Aubin C et al. Genetic Variation in the IL7RA/IL7 Pathway Increases Multiple Sclerosis Susceptibility. Hum Genet. 2010;127(5):525-535.

6. Weber F, Fontaine B, Cournu-Rebeix I, Kroner A, Knop M, Lutz S, et al. IL2RA and IL7RA genes confer susceptibility for multiple sclerosis in two independent European populations. Genes and immunity. 2008;9(3):259-63.

7. Ibayyan L, Zaza R, Dahbour S, El-Omar A, Samhouri B, El-Khateeb $\mathrm{M}$, et al. The promoter SNP, but not the alternative splicing SNP, is linked to multiple sclerosis among Jordanian patients. J Mol Neurosci. 2014;52(4):467-72.

8. Oksenberg JR, Baranzini SE, Sawcer S, Hauser SL. The genetics of multiple sclerosis: SNPs to pathways to pathogenesis. Nat Rev Genet. 2008;9(7):516-26.

9. McDonald WI, Compston A, Edan G, Goodkin D, Hartung HP, Lublin FD, et al. Recommended diagnostic criteria for multiple sclerosis: guidelines from the International Panel on the diagnosis of multiple sclerosis. Ann Neurol. 2001;50(1):121-7.

10. Kurtzke JF. Rating neurologic impairment in multiple sclerosis: an expanded disability status scale (EDSS). Neurology. 1983;33(11):1444-52.

11. Zhang R, Duan L, Jiang Y, Zhang X, Sun P, Li J, et al. Association between the IL7R T244I polymorphism and multiple sclerosis: a meta-analysis. Mol Biol Rep. 2011;38(8):5079-84. doi: 10.1007/ s11033-010-0654-5. 
12. Heidari M, Behmanesh M, Sahraian MA. Variation in SNPs of the IL7Ra gene is associated with multiple sclerosis in the Iranian population. Immunol Invest. 2011;40(3):279-89.

13. Traboulsee AL, Bernales CQ, Ross JP, Lee JD, Sadovnick AD, VilarinoGuell C. Genetic variants in IL2RA and IL7R affect multiple sclerosis disease risk and progression. Neurogenetics. 2014;15(3):165-9.
14. Pandit L, Ban M, Sawcer S, Singhal B, Nair S, Radhakrishnan K, et al. Evaluation of the established non-MHC multiple sclerosis loci in an Indian population. Mult Scler. 2011;17(2):139-43.

15. Gregory SG, Schmidt S, Seth P, Oksenberg JR, Hart J, Prokop A, et al. Interleukin 7 receptor alpha chain (IL7R) shows allelic and functional association with multiple sclerosis. Nat Genet. 2007;39(9):1083-91. 